\title{
NeuroImage
}

\section{Validating the independent components of neuroimaging time series via clustering and visualization}

\author{
Johan Himberg, ${ }^{a}$ Aapo Hyvärinen, ${ }^{b, *}$ and Fabrizio Esposito ${ }^{\mathrm{c}, \mathrm{d}}$ \\ ${ }^{a}$ Neural Networks Research Centre, Helsinki University of Technology, Helsinki, Finland \\ ${ }^{\mathrm{b}}$ Department of Computer Science, Helsinki Institute for Information Technology/BRU, University of Helsinki, Helsinki Finland \\ " Second Division of Neurology, Second University of Naples, "Federico II", Naples, Italy \\ 'Department of Neurological Sciences, University of Naples, "Federico II", Naples, Italy
}

Received 17 November 2003; revised 9 March 2004; accepted 11 March 2004

Recently, independent component analysis (ICA) has been widely used in the analysis of brain imaging data. An important problem with most ICA algorithms is, however, that they are stochastic; that is, their results may be somewhat different in different runs of the algorithm. Thus, the outputs of a single run of an ICA algorithm should be interpreted with some reserve, and further analysis of the algorithmic reliability of the components is needed. Moreover, as with any statistical method, the results are affected by the random sampling of the data, and some analysis of the statistical significance or reliability should be done as well. Here we present a method for assessing both the algorithmic and statistical reliability of estimated independent components. The method is based on running the ICA algorithm many times with slightly different conditions and visualizing the clustering structure of the obtained components in the signal space. In experiments with magnetoencephalographic (MEG) and functional magnetic resonance imaging (fMRI) data, the method was able to show that expected components are reliable; furthermore, it pointed out components whose interpretation was not obvious but whose reliability should incite the experimenter to investigate the underlying technical or physical phenomena. The method is implemented in a software package called Icasso.

(C) 2004 Elsevier Inc. All rights reserved.

Keywords: Clustering; Visualization; Neuroimaging time series

\section{Introduction}

Independent component analysis (ICA) (Hyvärinen et al., 2001b) is a general-purpose statistical model that has been widely used in the analysis of brain imaging data (see, e.g., Makeig et al., 1997; McKeown et al., 1998; Vigário et al., 2000). A major problem in application of ICA is that the reliability of the estimated independent components is not known. An ICA algo-

* Corresponding author. Department of Computer Science, PO Box 26, 00014 University of Helsinki, Helsinki, Finland. Fax: +358-9-191-44441.

E-mail addresses: johan.himberg@hut.fi (J. Himberg),

aapo.hyvarinen@helsinki.fi (A. Hyvärinen), faesposi@unina.it

(F. Esposito).

Available online on ScienceDirect (www.sciencedirect.com.) rithm gives a specified number of components, but it is not known which ones deserve further analysis and discussion.

As with any statistical method, it is necessary to analyze the statistical reliability (statistical significance) of the obtained components. The finite sample size induces statistical errors in the estimation - this is the case where classical analysis of statistical reliability should be used (Meinecke et al., 2002). Such an analysis can be accomplished using bootstrapping, a well-known computational method for computing the statistical reliability in the case where a simple mathematical formula cannot be found (Efron and Tibshirani, 1993). Bootstrapping is a resampling method; that is, the data sample is randomly changed by simulating the sampling process, and the algorithm is then run many times with the bootstrapped samples that are somewhat different from each other. The reliability of the original estimate can then be analyzed by looking at the spread of the obtained estimates.

A further problem typical of ICA is that most algorithms have random (stochastic) elements; that is, the algorithms give somewhat different results at every run of the algorithm. Most ICA algorithms such a FastICA (Hyvärinen, 1999) or the natural gradient (infomax) algorithm (Amari et al., 1996; Bell and Sejnowski, 1995) are based on minimization or maximization of an objective function, such as the likelihood, mutual information, or negentropy (see Hyvärinen and Oja, 2000; Hyvärinen et al., 2001b for a discussion). The randomness of the algorithm stems from the fact that it is not possible to find the point that globally minimizes the objective function (for simplicity of terminology, we shall talk about minimization only because the principle is the same in the case of maximization).

The problem is that most ICA algorithms are based on methods related to gradient descent. The basic principle is to start in some initial point, and then make steps in a direction that decreases the objective function, until one finds a point in which the objective function is locally minimized. Depending on the point where the search was started (the "initial condition"), the algorithm will find different local minima. In the case of a very high-dimensional signal space, the probability of finding the global minimum may be very small. Yet, in the case of a highdimensional signal space, the situation is even more complicated, 
because at each local minimum, a subset of the components may be accurately estimated. This is plausible because the estimation of the individual components is partly decoupled from each other; in fact, the components can be estimated one at a time by using the criterion of negentropy or nongaussianity (Hyvärinen, 1999). Thus, to assess the algorithmic reliability of the estimated components, it is reasonable to run the estimation algorithm many times, using different initial points, and assessing which of the components are found in almost every run.

Algorithmic and statistical reliabilities in the context of stochastic algorithms and local minima are illustrated in one dimension in Fig. 1. It is important to understand that these two reliabilities are quite distinct properties. What we call statistical reliability is basically the same as the classic notion of statistical significance. Algorithmic reliability, on the other hand, is a phenomenon that is irrelevant in the case of most classic statistical methods; problems only appear in connection with modern computationally intensive methods such ICA.

In this paper, we present a tool for investigating the reliability of the independent components. The method is based on estimating a large number of candidate independent components by running an ICA algorithm many times, and visualizing their clustering in the signal space. Each estimated independent component is one point in the signal space. If an independent component is reliable, (almost) every run of the algorithm should produce one point in the signal space that is very close to the "real" component. Thus, reliable independent components correspond to clusters that are small and well separated from the rest of the estimates. In contrast, unreliable components correspond to points which do not belong to any cluster. We investigate both algorithmic and statistical reliability by running the ICA algorithm either with different initial conditions or with different bootstrapped data sets, respectively.

Our focus is on constructing a comprehensive set of methods supported by explorative data analysis and visualization. We have a)

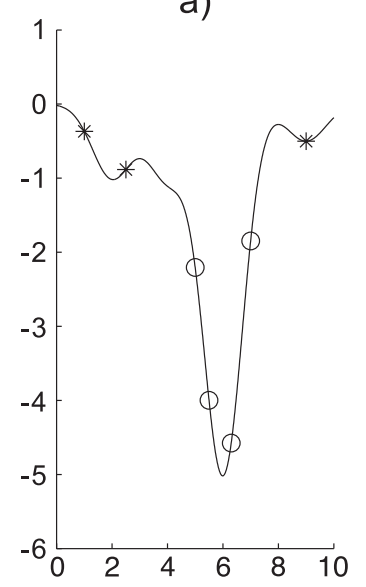

b)

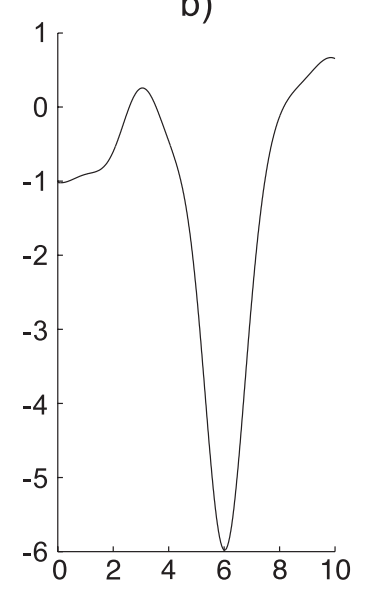

Fig. 1. Illustration of the problem of local minima. (a) An objective function that is to be minimized. There is a global minimum at 6 , and small local minima at 2 and 9 . If a gradient descent algorithm starts at the points marked by circles, it will probably find the global minimum. However, if it starts at the points marked by stars, it will probably converge the nearest local minimum, at 2 or 9 . Running the algorithm many times, it will converge to 6 most of the time. (b) When the data is resampled in the bootstrapping method, the optimized function changes a bit. The smaller local minima at 2 and 9 disappear, and a new local minimum appears at 0 . The stable minimum at 6 is still a local (and global) minimum. developed a software package called Icasso $^{1}$ to implement these operations and visualize the results. Preliminary results were reported in Himberg and Hyvärinen (2003).

\section{The Icasso software package}

\section{Overview of Icasso}

First, we provide a concise overview of our method for ICA reliability analysis, Icasso. It consists of the following steps:

(1) Parameters for the ICA estimation algorithm are selected. We use FastICA whose parameters include the orthogonalization approach (symmetric or deflationary), the nonlinearity, etc.

(2) The ICA algorithm is run a number of times using the selected training parameters. Each time, the data is bootstrapped and/or the initial point of the optimization is changed.

(3) The estimates (estimated components) are clustered according to their mutual similarities. In principle, the clustering method can be freely selected. We apply agglomerative clustering with average-linkage criterion.

(4) The clustering is visualized as a 2-D plot. The user investigates how the estimates are concentrated in the clusters. The clustering of the estimated components is expected to yield information on the reliability of estimation. A compact, tight cluster emerges when a similar estimate is repeatedly obtained despite the randomization.

(5) The user can retrieve the estimates belonging to certain cluster(s) for further analysis and visualization.

To complete steps 1-3, the user simply sets the FastICA parameters and launches a resampling and clustering application. In step 4, the user explores the clustering by launching an interactive visualization application. The user can examine the quality of the clusters and rank them accordingly. Subsequently, Icasso visualizes the similarity matrix between all the estimates and their partition into clusters in a single graph. Thus, the user can examine relationships between estimates and clusters in detail. In step 5, the user can retrieve any set of estimates that belong to certain cluster(s).

Our criteria for selecting the specific estimation and visualization methods for Icasso were that (i) methods for completing each subtask are well known, (ii) they support visualization and explorative data analysis, and (iii) to avoid redundant work, existing, publicly available building blocks should be used. ${ }^{2}$ In the rest of this section, we provide a detailed description of the method.

\section{Generating the estimates and comparing them}

We consider the standard linear, noise-free ICA model $\mathbf{x}=\mathbf{A s}$ of independent sources $\mathbf{s}$ and a mixing matrix A. However, what is usually estimated in practice, is the demixing matrix $\mathbf{W}$ for $\mathbf{s}=\mathbf{W} \mathbf{x}$, where $\mathbf{W}$ is a (pseudo)inverse of $\mathbf{A}$ (Hyvärinen et al., 2001b).

\footnotetext{
1 The MATLAB package is available at http://www.cis.hut.fi/projects/ ica/icasso.

2 We use the FastICA Toolbox 2.1 and the SOM Toolbox 2.0 (Vesanto et al., 2000) for MATLAB, both freely available from http:/www.cis.hut.fi/ research/software.shtml.
} 
The FastICA algorithm is run $M$ times on the data matrix $\mathbf{X}=$ $\left[\begin{array}{lll}\mathbf{x}_{1} & \mathbf{x}_{2} \ldots \mathbf{x}_{N}\end{array}\right]$ consisting of $N$ samples of $k$-dimensional vectors. The estimates of demixing matrices $\hat{\mathbf{W}}_{i}$ from each run $i=1,2, \ldots, M$ are collected into a single matrix $\hat{\mathbf{W}}=\left[\hat{\mathbf{W}}_{1}^{\mathrm{T}} \hat{\mathbf{W}}_{2}^{\mathrm{T}} \ldots \hat{\mathbf{W}}_{M}^{\mathrm{T}}\right]^{\mathrm{T}}$. If $n_{i}$ independent components are estimated on each round, we get $K=$ $\sum_{i} n_{i}$ estimates, and the size of $\hat{\mathbf{W}}$ will be $K \times k$.

We can recompute independent component estimates by (a) Randomizing the initial condition: FastICA is run $M$ times for the same data $\mathbf{X}$, so that for each run the algorithm starts from a new random initial condition; (b) Bootstrapping: FastICA is run $M$ times. The initial condition is kept the same in every run, but the data is resampled by bootstrapping every time; and (c) Bootstrapping with randomized initial condition as a combination of (a) and (b).

A natural measure of similarity between the estimated independent components is the absolute value of their mutual correlation coefficients $r_{i j}, i, j=1, \ldots, K$. Straightforward calculations show that they can be efficiently computed by simple matrix multiplication. In fact, each correlation is given by one element of the matrix $\mathbf{R}=\hat{\mathbf{W}} \mathbf{C} \hat{\mathbf{W}}^{\mathrm{T}}$ where $\mathbf{C}$ is the covariance matrix of the original data $\mathbf{X}$ (this is exactly true in the case of different initial points; for bootstrapping, some extra normalization is necessary). The final similarity matrix then has the elements $\sigma_{i j}$ defined by

$\sigma_{i j}=\left|r_{i j}\right|$.

Later, we use clustering methods and validity indices that use dissimilarities (distances). Therefore, we need to transform the similarity matrix into a dissimilarity matrix with elements $d_{i j}$. A classic way to make this transformation is obviously given by (Everitt, 1993):

$d_{i j}=1-\sigma_{i j}$.

We will also use a simple modification of this formula as will be explained below.

\section{Clustering the estimates}

We can partition the set of all estimates (estimated independent components) into several disjoint clusters using some basic clustering algorithm and the dissimilarity measure in Eq. (2). Agglomerative hierarchical clustering is a well-known method for a modest number of objects (Everitt, 1993; Gordon, 1987)). The tree-like hierarchy (dendrogram) produced by agglomeration is intuitively appealing in the sense that all clusters implied by lower levels of the tree are always subsets of clusters at the higher levels. Thus, the user is able to explore and compare the different level(s) of clustering that are readily computed. The obvious way to obtain a partition of $L$ clusters from a dendrogram is to cut it at level where $L$ clusters are present. A dendrogram is illustrated in Fig. 2.

There are numerous reviews and studies on the many agglomeration strategies and cluster validity indices (see, e.g., Bandyopadhay and Maulik, 2001; Bezdek and Pal, 1998; Everitt, 1993; Gordon, 1987; Maulik and Bandyopadhay, 2002). Unfortunately, there is no easy way of selecting the optimal agglomeration strategy for a specific set of data, and the selection must be based on problem-specific considerations. The same applies also to selecting a clustering validity index for determining a "natural" number of clusters (Bezdek and Pal, 1998).

Three basic agglomeration strategies that operate directly on the similarity matrix are single-link (SL), complete-link (CL), and

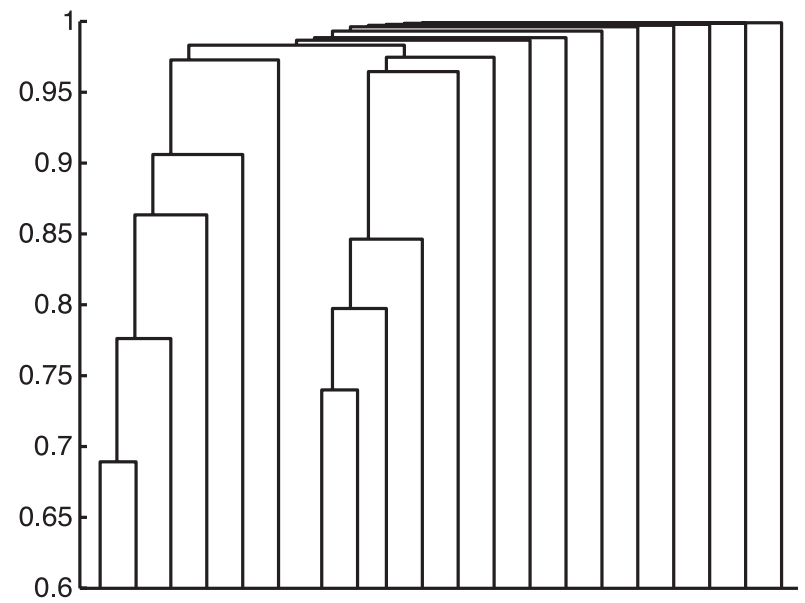

Fig. 2. An illustration of a dendrogram and hierarchical agglomerative clustering. The points are successively joined into clusters when moving upwards in the dendrogram. The vertical axis gives the dissimilarity for which the clusters are merged. Clustering can be performed at any level.

group average-link (AL). Icasso uses $\mathrm{AL}$ as the default choice of agglomeration strategy. This is because, first, SL is in general reported to be more sensitive to noise than AL and CL (Everitt, 1993). Second, our experiments (not shown) revealed that when the number of clusters is smaller than the data dimension $(L<k)$, CL starts to join clusters inconsistently.

To direct the attention of the user to those clusters that seem to be the most compact and interesting, we introduce a (conservative) cluster quality index $I_{\mathrm{q}}$ that reflects the compactness and isolation of a cluster. It is computed as the difference between the average intracluster similarities and average intercluster similarities. Let us denote by $C$ the set of indices of all the estimated components, by $C_{m}$ the set of indices that belong to the $m$ th cluster and by $\left|C_{m}\right|$ the size of the $m$ th cluster. Then, we define the cluster quality index as

$I_{\mathrm{q}}\left(C_{m}\right)=\frac{1}{\left|C_{m}\right|^{2}} \sum_{i, j \in C_{m}} \sigma_{i j}-\frac{1}{\left|C_{m} \| C_{-m}\right|} \sum_{i \in C_{m}} \sum_{j \in C_{-m}} \sigma_{i j}$,

where $C_{-m}$ is the set of indices that do not belong to the $m$ th cluster. Eventually, $I_{\mathrm{q}}\left(C_{m}\right)$ is equal to one for an ideal cluster when Eq. (1) is used to compute the similarities $\sigma_{i j}$, and decreases when $C_{m}$ becomes less compact and isolated.

An important parameter that needs to be selected in any clustering method is the number of clusters $L$. We prefer leaving the final selection of the number of clusters to the user who can interactively explore the results produced by different levels of dendrogram. It is reasonable to start studying the clustering from the number of clusters $L$ equal to the data dimension $k$ and investigate the values of cluster quality index in rank order, as will be done in Fig. 5 .

It is also possible to use quantitative indices for suggesting the number of clusters that best fits to the data. We considered some of the cluster validity indices that can be computed knowing only the dissimilarity matrix. These included four of the Dunn-like indices in Bezdek and Pal (1998) and the $R$-index defined in Levine and Domany (2001). Empirical studies on such indices (e.g., Bandyopadhay and Maulik, 2001; Bezdek and Pal, 1998; Maulik and Bandyopadhay, 2002) often yield different results depending on the character of the data used, with no clear indication of general superiority. Our own experiments on these indices did not suggest 


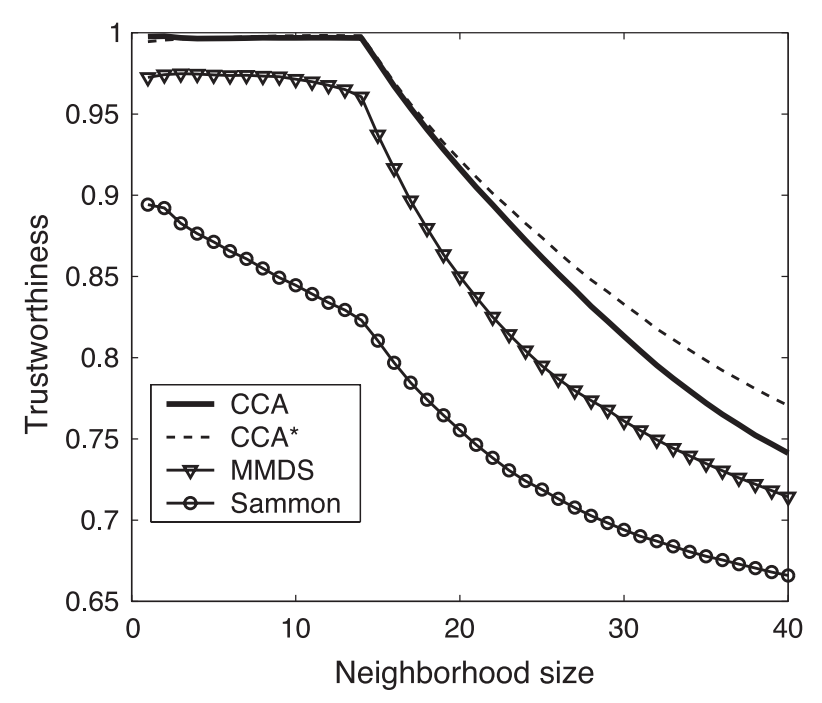

Fig. 3. Comparison of different projection methods. The trustworthiness of CCA, Sammon's projection and MMDS are plotted as a function of neighborhood size $(1-40)$. CCA* is obtained when Eq. (2) was used, the rest of the graphs result from using Eq. (5). Curvilinear component analysis has the highest values of trustworthiness for all neighborhood sizes.

any definitive winner, either. Currently, Icasso shows the $R$-index $\left(I_{R}\right)$ in the user interface. The index is defined as

$I_{R}=\frac{1}{L} \sum_{m=1}^{L} \frac{S_{m}^{\mathrm{in}}}{S_{m}^{\mathrm{ex}}}$

where

$S_{m}^{\text {in }}=\frac{1}{\left|C_{m}\right|^{2}} \sum_{i, j \in C_{m}} d_{i j}$, and $S_{m}^{\mathrm{ex}}=\min _{m \neq m} \frac{1}{\left|C_{m} \| C_{m}^{\prime}\right|} \sum_{i \in C_{m}} \sum_{j \in C_{m}^{\prime}} d_{i j}$.

The index is a variant of the Davies-Bouldin index (see Bezdek and Pal, 1998). It searches for compact and well-separated clusters and the minimum of $I_{R}$ suggests the best partition. However, we note that such an index should be used only in connection with explorative investigation.

As a spin-off of the reliability analysis, Icasso is also able to improve the estimates of the components. In fact, it is possible to integrate information over the many runs performed by computing a representative point for each tight cluster. Icasso provides the "centrotype" of the cluster as such a representative point. The centrotype is the point in the cluster that has the maximum sum of similarities (as measured by correlation coefficients) to other points in the cluster.

\section{Visualization by nonlinear 2-D projection}

In addition to assigning the estimated components to clusters, Icasso provides a tool for getting a detailed look into the clustering results, including relations between the clusters and the individual estimates. The result of the hierarchical clustering is typically presented as a dendrogram, but other types of visualization also exist. In Icasso, each estimate is plotted as a point on the display, and a convex hull bounds the estimates belonging to the same cluster (Gordon, 1987). This presentation allows visualizing the similarities $\sigma_{i j}$ rather explicitly; the points are connected with lines whose thickness/color represent the similarities between them (see below).
We apply projection methods related to multidimensional scaling (MDS) as suggested in Gordon (1987) to approximate the original dissimilarities between estimates by Euclidean distances in two dimensions. This should result in a projection where the smaller a convex hull is, the more compact the corresponding cluster is. An ideal cluster should contract into a single point.

For this purpose, we compared the linear metric MDS (MMDS) (Torgerson, 1952), and two nonlinear methods: Sammon's projection (Sammon, 1969) and Curvilinear Component Analysis (CCA) (Demartines and Hérault, 1997). In addition to visual comparison, we used a trustworthiness index proposed in Venna and Kaski (2001). Spatial proximity is one of the strongest visual indicators of grouping (Ware, 2000). To be trustworthy, a projection should be such that one can trust the visual proximity as an indicator of similarity. The trustworthiness index in Venna and Kaski (2001) is a function of the neighborhood size, and it must be evaluated for the neighborhood sizes of interest; according to Venna and Kaski (2001), it is especially important that the trustworthiness is retained for small neighborhoods.

According to our experiments (see Fig. 3), CCA produces more trustworthy projections than MMDS or Sammon's method for the dissimilarity measure in Eq. (2). We also considered visualization based on the Self-Organizing Map (SOM) since it is reported to be more trustworthy than many MDS-like methods (Nikkilä et al., 2002; Venna and Kaski, 2001). However, we abandoned this method because its regular grid visualization forces the lines of the similarity graph to shadow each other more than they do on a nonuniform projection.

The projection can be further controlled by modifying the definition of dissimilarity in Eq. (2) suitably, for example, as

$$
d_{i j}^{*}=\sqrt{1-\sigma_{i j}}
$$

This spreads the distribution of the distances so that differences in size among the most compact clusters can be seen better. For this reason, Icasso uses transformation in Eq. (5) instead that of Eq. (2) for making the visualization.

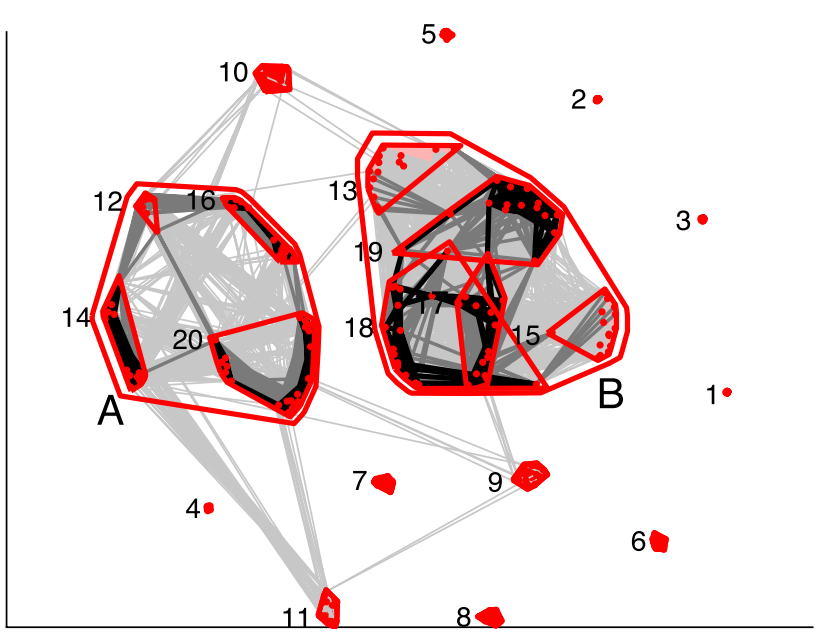

Fig. 4. The similarity graph of the estimates for MEG data. Clusters are indicated by red convex hulls. Grey lines connect estimates whose similarity is larger than a threshold, the darker the line the stronger the similarity. Labels 1-20 correspond to Fig. 5. Convex hulls A and B show how clusters agglomerate further if the number of clusters is set to $L=13$ instead of $L=20$. 
The projection onto the $2 \mathrm{D}$ plane usually contains some distortions, and therefore, it is useful to show some similarities (correlations) by additional lines connecting the points. Such lines are drawn in different colours between dots representing estimates whose correlation (in absolute value) exceeds a certain threshold. These thresholds are specified by the user; here we report the thresholds used in Fig. 4. Icasso draws the lines in three different gray levels, here using light-grey lines between estimates whose correlation (in absolute value) is larger that 0.1 ; midgrey lines are drawn for $|r|>0.58$ and black lines for $|r|>0.82$. To reduce the number of graph lines, clusters that have an average within-cluster larger than 0.9 are painted with solid light red and no lines are shown within the cluster; clusters are painted with bright red if the minimum within-cluster $|r|$ is larger than 0.9.

\section{Illustrative experiments}

Here we illustrate the utility of Icasso by reanalyzing two data sets whose properties are well known to us. First, we experimented with a magnetoencephalographic (MEG) data set described in more detail in Vigário et al. (1998). Next, we applied Icasso on functional magnetic resonance imaging (fMRI) data from a finger-tapping experiment, described in more detail in Esposito et al. (2003).

\section{Magnetoencephalographic data}

\section{Methods}

The data consisted of preprocessed signals originating from 122-channel whole-scalp MEG measurements from the brain (Vigário et al., 1998). The output of each sensor was considered one mixture signal. The original signals were band-pass filtered between 0.5 and $45 \mathrm{~Hz}$, and the data dimension $(k)$ was reduced from 122 to 20 using principal component analysis (PCA) to reduce noise and overlearning. The recording lasted about 2 min and contained 17,730 samples. The measurements from the brain were disturbed by signals originating from various sources: heart beat, eye blinks and saccade, other muscular activity — and a digital watch.

We ran Icasso five times using three different settings. Setting (I) random initial conditions, third power as nonlinearity; (II) as I, but hyperbolic tangent (tanh) as the nonlinearity; and (III) as I, but using both bootstrapping and random initial conditions.

Each time number of randomizations $(M)$ was 15 , and the symmetrical approach was used in FastICA.

\section{Results and discussion}

In the following, we present results for a particular test run from setting I. First, we select the number of clusters $L$ to equal 20, which is the dimension of the data (after PCA). The clusters and their interrelation are visualized in Fig. 4. Fig. 5 shows the quality index $I_{\mathrm{q}}$ for each cluster. Note how the diameter of the convex hulls representing the clusters grows when the value of quality index $I_{\mathrm{q}}$ decreases.

We notice a "knee" in the graph presenting the ranked $I_{\mathrm{q}}$ when moving from cluster nos. 10 to 12 in Fig. 5. In addition, the clustering validity index $I_{R}$ has a local minimum for $L=13$ (not shown). Thus, it is interesting to consider the case $L=13$. Convex hulls marked A and B show how clusters are merged if $L=13$ is selected instead. The estimated source signals for centrotypes

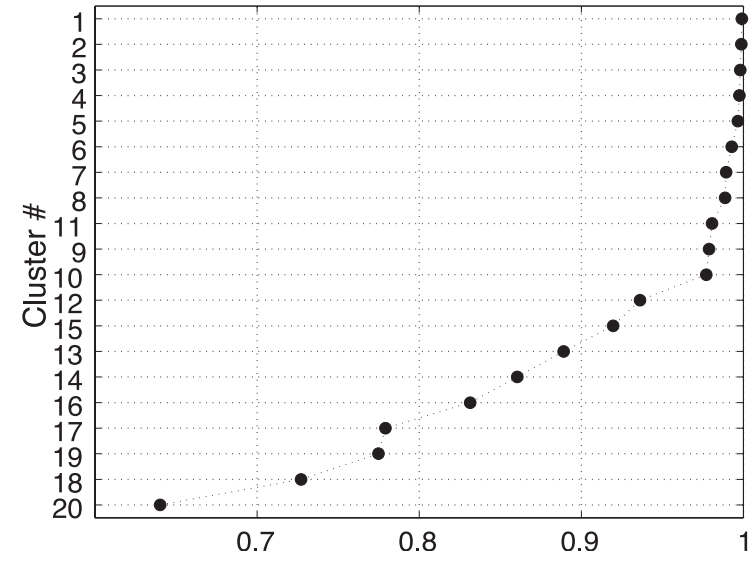

Fig. 5. The quality index $I_{\mathrm{q}}$ in rank order for the clusters in Fig. 4.

associated to the most tightest clusters \#1-11 (being outside of convex hulls A and B), are presented in quality rank order in Fig. 6. From the previous studies, we know that source estimates \#1 and \#2 correspond to eye movements, \#3 to heart and \#7 to the digital watch. Sources $\# 5$ and $\# 6$ are related to muscular activities due to biting. Thus, well-known strong artifacts are all ranked to the top which is quite reasonable. In repeated runs of Icasso with different settings, all top four estimates were always ranked 1-4 with the first and the second only occasionally changing places. The next seven estimates remained usually in top 11, except that estimates \#5-6 related to biting became less reliably estimated, especially in setting II.

Icasso is thus able to point out some components that are worth investigating further. Above all, source \#4 is interesting since it is clearly well estimated - even in repeated experiments and in other settings - but the physiological explanation, if any, is not yet known. The same applies, to a lesser extent, to the components $\# 8-11$. These components may not look very interesting at first sight, which is possibly why they were not investigated in detail by Vigário et al. (1998).

\section{Functional magnetic resonance images}

\section{Methods}

A healthy volunteer participated in a dominant-hand fingertapping fMRI experiment (Esposito et al., 2003). Images were acquired on a 1.5-T superconducting SIGNA MR scanner (General Electric Medical Systems, Milwaukee, WI, USA) using a standard circularly polarized head coil. T1-weighted structural volumes served as anatomical reference for the positioning of five slices parallel to the bicommissural plane to cover optimally the primary motor and supplementary motor areas. The functional scans were acquired using a conventional gradient-echo echoplanar imaging sequence (TR, $2 \mathrm{~s}$; echo time TE, $60 \mathrm{~ms}$; delay time, $2 \mathrm{~s}$ thus resulting in the effective TR of $4000 \mathrm{~ms}$; flip angle 90, field of view $210 \mathrm{~mm}$, matrix 128128, slice thickness $5 \mathrm{~mm}$, slice gap $2 \mathrm{~mm}$ ).

The experimental paradigm consisted of five intervals of five time points during which a self-paced finger-tapping task (sequential opposition of all fingers of the right-hand against the thumb) at a specified frequency of $2 \mathrm{~Hz}$ was carried out, alternated with five intervals of five time points of resting. The alternation between task and rest conditions was verbally trig- 
gered. Although the tapping rate was self-paced and the tapping force unconstrained, the motor performance was carefully monitored for being of constant rate and force during the experimental periods. An additional "rest" block was acquired before the first "task" block of which the first two time points were discarded to avoid the global effects induced by incomplete magnetization equilibrium.

Each image of the slice time series was smoothed in space by applying an isotropic gaussian kernel to low-pass filter each slice at each time point. Then, the dimensionality of the data was reduced to 30 by PCA. ICA can be performed on fMRI data is two different ways, called spatial and temporal ICA (Calhoun et al., 2001b); here we used spatial ICA. In FastICA, we used a symmetric approach, the nonlinearity being tanh. We tested the algorithmic reliability by running the ICA algorithm 15 times with random initial conditions.

\section{Results and discussion}

The similarity graph is shown in Fig. 7, and the five first centrotype activation maps are shown in Fig. 8. Based on previous studies, we interpret the first five clusters as follows:

1. Task-related source. The highest values of the component clearly appear to cluster anatomically in contralateral rolandic region of the primary motor cortex and the supplementary motor area. The primary somatosensory area (SI) is active as well.

2. Vascular source, showing activation foci that cluster in the regions of large blood vessels. The time course (not shown) was poorly related to task execution.

3. This source is difficult to interpret and worthy of further study. The nice bilateral structure and the shape of the time course suggest the classification of this source as an "auditory source" caused by the auditory cue (GO-STOP), but it is not possible to confirm this hypothesis in the absence of more inferior slices that covered primary and secondary cortices.

4. Motion-related source, active at the boundaries of brain (typical ring-like activation patterns). These phenomena are representative of head motion effects during scanning. The associated time course (not shown) exhibited a long-term effect on the data, starting at the onset of the first task block.

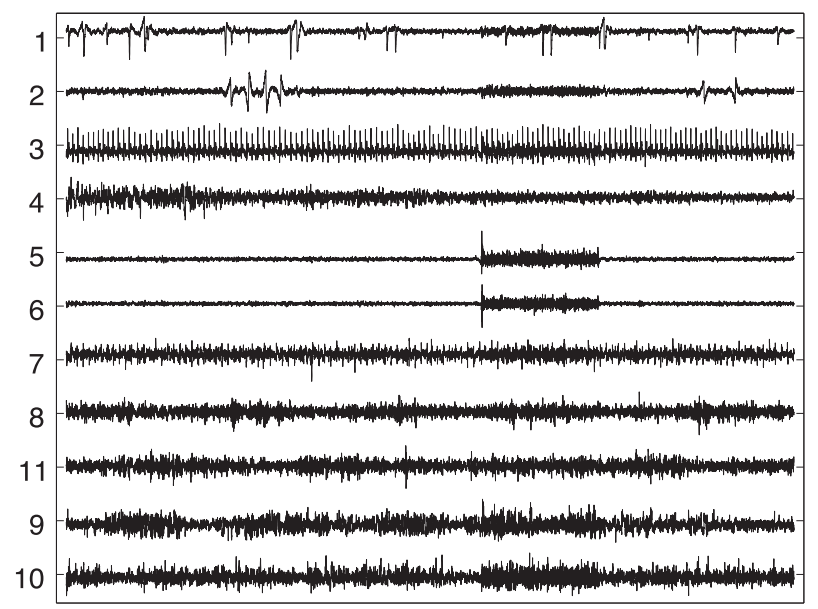

Fig. 6. Estimated sources corresponding to centrotypes of clusters \#1-11 in Fig. 4.

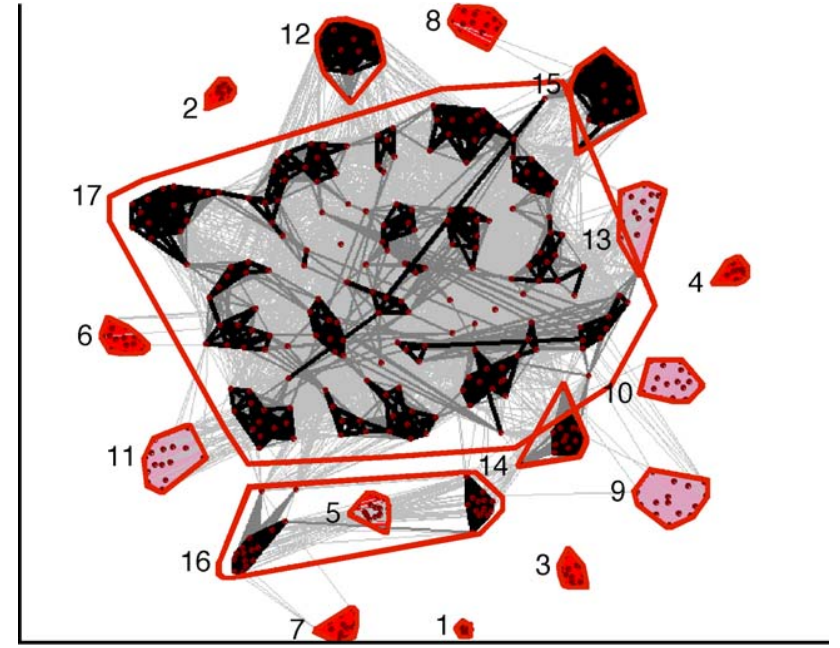

Fig. 7. The similarity graph of the estimates for fMRI data. Clusters are indicated by red convex hulls and grey/black lines connect similar estimates.

5. Another vascular source, showing activation foci that cluster in the regions of large blood vessels. This source exhibits an evident correlation with the task and includes the motor cortex activity. Possibly, it corresponds to modulation of task-related activity.

Thus, again we see that in addition to showing that some wellknown components are reliable (here, in the algorithmic sense), Icasso is able to point out a component (\#3) whose interpretation is not obvious and which might not capture the attention of the experimenter who just runs ICA once and is confronted with a large number of estimated components.

\section{Discussion}

\section{Utility of reliability analysis}

We have shown how the reliability of the independent components can be analyzed by a new software package. On one hand, it is obvious why reliability analysis is needed; any conclusions on the data should be based only on components that have been found reliable. On the other hand, reliability analysis provides an interesting data "mining" tool in itself; it highlights some components and suggests that the experimenter discard some others. This may greatly reduce the amount of work in the analysis of the components given by an ICA algorithm because there is now extra information on which components are worthy of further study. This information is objective and reduces the subjective element that is typically present in the selection of "interesting" components among the dozens given by ICA. In fact, we saw in both sets of experiments above that Icasso is able to find some components that may not look interesting to the typical experimenter but which are highly reliable.

\section{Related work}

Meinecke et al. (2002) have previously proposed a method for analyzing the statistical reliability of independent components. It 

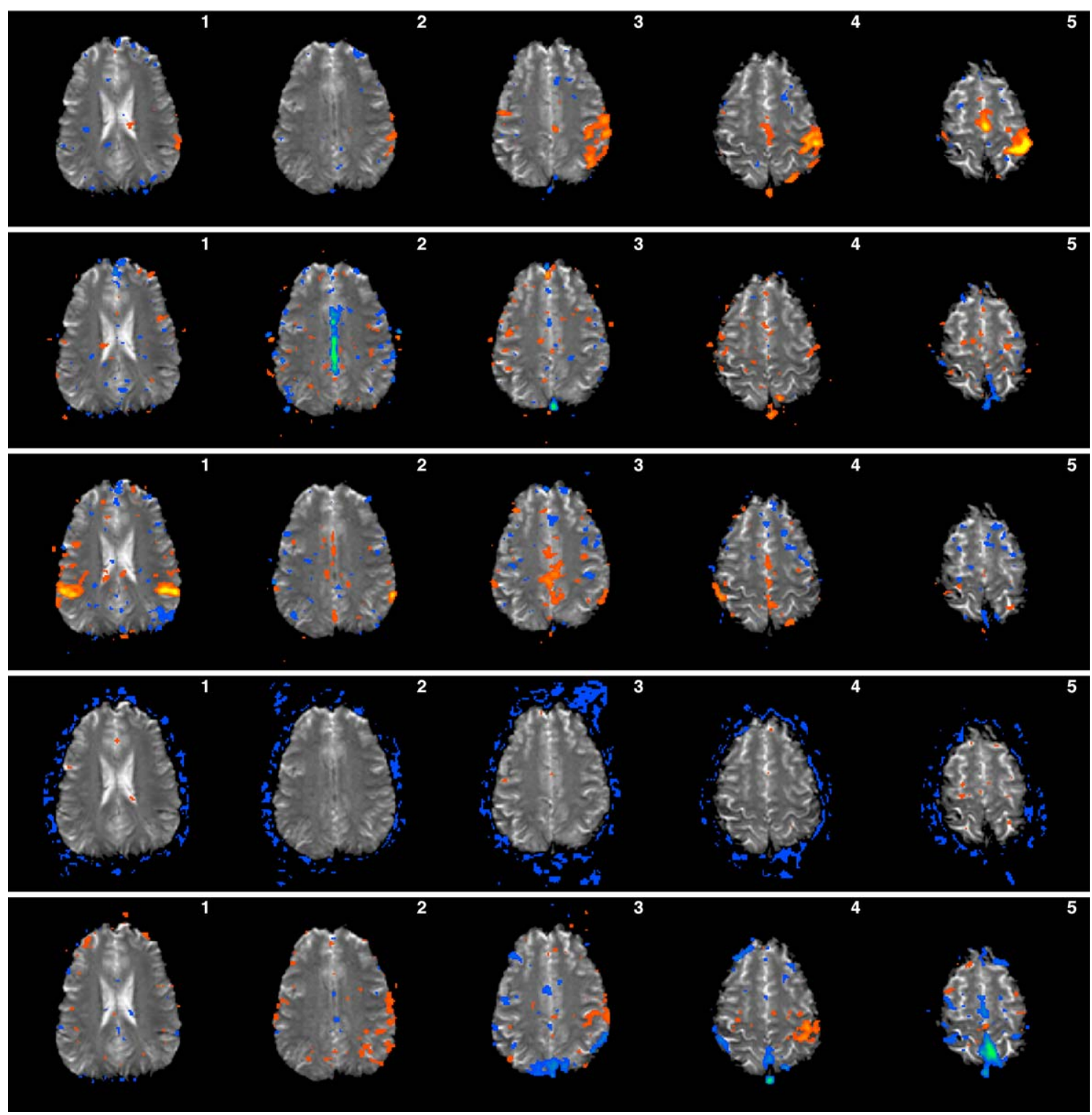

Fig. 8. The centrotypes of the five best clusters (top is best) in the fMRI experiment.

seems that their method is mainly applicable in the case of algorithms that are algorithmically very reliable, which is not the case for the algorithms used in most analyses of independent components of brain imaging data. This is because Meinecke et al. (2002) assumed that a small perturbation in the data results in a small perturbation in the estimated mixing matrix, which is not always true for the most widely used ICA algorithms where a small perturbation in the data (or the initial conditions) may make the estimator go to a different local minimum. Another difference to our approach is that Meinecke et al. (2002) did not analyze the algorithmic reliability of the estimates. Related studies on validating components estimated from brain imaging data can be found in Calhoun et al. (2001a) and Duann et al. (2003).

An alternative approach to ours would be to measure the independence of the components given by the algorithm. Basically, as most ICA algorithms are based on the maximization of some measure of independence (Hyvärinen et al., 2001b), this boils down to measuring how well the algorithm optimized its objective function. Thus, we could run the ICA algorithm many times and choose the results of the run that gave the most independent components. Such an approach seems to be quite unsatisfactory, however. Some of the components may be well estimated in some runs, others in other runs, so it is not reasonable to assume that the best run gives the best estimates for each component. In Icasso, we are able to combine information from several runs of the algorithms. Indeed, we thus obtain a set of components (given by the cluster centers in the signal space) that is presumably better than any of the component sets provided by a single run. This is because it is likely that the errors in the different estimates are independent from each other; thus, the average of many estimates should have a smaller error because the errors are reduced by averaging.

Let us also mention some further methods that may be of help in finding the most interesting or meaningful components given by ICA. Formisano et al (2002) developed some indices that are correlated with the "interestingness" of the components; the components can be ordered using these indices after ICA estima- 
tion. Hyvärinen et al. (2001a) developed a method that orders components on a one-dimensional line or a two-dimensional grid so that components that belong together, possibly being manifestations of the same multidimensional signal source, are close to each other.

\section{Future directions}

As for the computational load of the algorithm, we have noted that the hierarchical clustering is clearly the bottleneck in the current computational environment. Currently, say, 6001000 estimated components can be handled on an ordinary PC, which corresponds to, for example, 10 runs of the algorithm when the dimension of the data is 60-100. On the current implementation the computational load in terms of time consumption rises as a cubic function of the number of estimates. Consequently, for bigger amounts of estimates, a more sophisticated implementation may be needed. We are currently investigating alternative methods for clustering.

A large number of components also make it difficult to find a satisfactory projection onto a two-dimensional plane. Thus, if the number of components greatly exceeds 100, the quality of the visualization suffers and more sophisticated methods may be necessary.

In fact, an important point that is ubiquitous in the application of ICA is choosing the number of principal components that are retained, which typically determines the number of independent components. In general, dimension reduction must be carefully considered since interesting components may present part of their variance in the low-power region of the eigenspectrum (Hyvärinen et al., 2001b), and may be lost in PCA. In principle, Icasso can be applied without any dimension reduction-with the reservations just given. In practice, however, the quality of the ICA results is greatly improved by PCA because the noise level is reduced. Choosing the actual number of components is usually done heuristically, which was also the case in this paper, although attempts at finding principled methods for determining the dimension have been made (see Hyvärinen et al., 2001b for some references). To control for the effect of PCA, we did another experiment with the MEG data taking a larger number of dimensions, 40 (results not shown). The stable components were essentially unchanged.

The present version of Icasso uses the FastICA algorithm, but it is possible to embed almost any ICA algorithm in the system. Speed is, however, a crucial factor because we have to run the ICA algorithm many times, which is why FastICA is very suitable for this purpose.

Finding clusters in the high-dimensional signal space involves fixing the number of clusters to be modeled, as well as the values of other internal parameters. Automatic determination of optimal values for these parameters is a most difficult theoretical problem; ultimately, the optimal values also depend on application-specific and subjective considerations. Therefore, we propose an interactive method based on visualization of the clustering structure. Yet, it may be possible to find purely automatic methods that offer satisfactory performance. Then the visualization step could be omitted, and numbers describing the reliability of each component could be automatically computed. At this point, it seems, however, that the visualization gives the user useful additional information on the structure of the clusters and may be quite valuable in its own right.

\section{Conclusions}

We have developed an interactive visualization method and software package for analyzing the reliability (both algorithmic and statistical) of independent components of brain imaging data. The basic principle is to run an ICA algorithm many times, and look at the clustering of the estimated components in the signal space. Each reliable component should produce a "tight" cluster of estimated components that are very close to each other and well separated from the rest. Reliability has two aspects, algorithmic and statistical, which can be probed by running the algorithm with different initial conditions or bootstrap samples, respectively.

\section{Acknowledgments}

A.H. was funded by the Academy of Finland, Research Fellow position and project \#48593. J.H. was partly supported by a Nokia Scholarship Grant from Nokia Foundation. We would like to thank Elia Formisano, Rainer Goebel, and Vince Calhoun for interesting discussions.

\section{References}

Amari, S.-I., Cichocki, A., Yang, H., 1996. A new learning algorithm for blind source separation. Adv. Neural Inf. Process. Syst., vol. 8. MIT Press, Cambridge, MA, pp. 757-763.

Bandyopadhay, S., Maulik, U., 2001. Nonparametric genetic clustering: comparison of validity indices. IEEE Trans. Syst. Man Cybern., Part C Appl. Rev. 31 (1), 120-125.

Bell, A., Sejnowski, T., 1995. An information-maximization approach to blind separation and blind deconvolution. Neural Comput. 7, $1129-1159$.

Bezdek, J., Pal, N., 1998. Some new indexes of cluster validity. IEEE Trans. Syst. Man Cybern., Part B, Cybern. 28, 301-315.

Calhoun, V., Adali, T., Pearlson, G., 2001a. Independent components analysis applied to fMRI data: a generative model for validating results. Proc. IEEE Workshop on Neural Networks for Signal Processing (NNSP'98), Falmouth, Massachusetts.

Calhoun, V., Adali, T., Pearlson, G., Pekar, J., 2001b. Spatial and temporal independent component analysis of functional MRI data containing a pair of task-related waveforms. Hum. Brain Mapp. 13, 43-53.

Demartines, P., Hérault, J., 1997. Curvilinear component analysis: a selforganizing neural network for nonlinear mapping of data sets. IEEE Trans. Neural Netw. 8 (1), 148-154.

Duann, J.-R., Jung, T.-P., Sejnowski, T., Makeig, S., 2003. What is consistent in ICA analysis of fMRI data? Stability assessment by repeated decomposition. Int. Symposium on Independent Component Analysis and Blind Signal Separation, pp. 289-294. Nara, Japan.

Efron, B., Tibshirani, R., 1993. An Introduction to the Bootstrap Chapman $\&$ Hall, New York.

Esposito, F., Seifritz, E., Formisano, E., Morrone, R., Scarabino, T., Tedeschi, G., Cirillo, S., Goebel, R., Di Salle, F., 2003. Real-time independent component analysis of functional MRI time-series. NeuroImage 20 (4), 2209-2224.

Everitt, B., 1993. Cluster Analysis, third ed. Edward Arnold, London.

Formisano, E., Esposito, F., Kriegeskorte, N., Tedeschi, G., Salle, F.D., Goebel, R., 2002. Spatial independent component analysis of functional magnetic resonance imaging time-series: characterization of the cortical components. Neurocomputing 49 (1-4), 241-254.

Gordon, A., 1987. A review of hierarchical classification. J. R. Stat. Soc., A $150(2), 119-137$.

Himberg, J., Hyvärinen, A., 2003. Icasso: software for investigating the 
reliability of ICA estimates by clustering and visualization. Proc. IEEE Workshop on Neural Networks for Signal Processing (NNSP2003). IEEE Press, Toulouse, France, pp. 259-268.

Hyvärinen, A., 1999. Fast and robust fixed-point algorithms for independent component analysis. IEEE Trans. Neural Netw. 10 (3), 626-634.

Hyvärinen, A., Oja, E., 2000. Independent component analysis: algorithms and applications. Neural Netw. 13 (4-5), 411-430.

Hyvärinen, A., Hoyer, P.O., Inki, M., 2001a. Topographic independent component analysis. Neural Comput. 13 (7), 1527-1558.

Hyvärinen, A., Karhunen, J., Oja, E., 2001b. Independent Component Analysis. Wiley, New York.

Levine, E., Domany, E., 2001. Resampling method for unsupervised estimation of cluster validity. Neural Comput. 13 (11), 2573-2593.

Makeig, S., Jung, T.-P., Bell, A.J., Ghahramani, D., Sejnowski, T., 1997. Blind separation of auditory event-related brain responses into independent components. Proc. Natl. Acad. Sci. U. S. A. 94, pp. $10979-10984$.

Maulik, U., Bandyopadhay, S., 2002. Performance evaluation of some clustering algorithms and validity indices. IEEE Trans. on Pattern Analysis and Machine Intelligence 24 (12), 1650-1654.

McKeown, M., Makeig, S., Brown, S., Jung, T.-P., Kindermann, S., Bell, A., Iragui, V., Sejnowski, T., 1998. Blind separation of functional magnetic resonance imaging (fMRI) data. Hum. Brain Mapp. 6 (5-6), $368-372$.

Meinecke, F., Ziehe, A., Kawanabe, M., Müller, K.-R., 2002. A resampling approach to estimate the stability of one-dimensional or multidimensional independent components. IEEE Trans. Biomed. Eng. 49 (12), $1514-1525$.

Nikkilä, J., Törönen, P., Kaski, S., Venna, J., Castrén, E., Wong, G., 2002. Analysis and visualization of gene expression data using self-organizing maps. Neural Netw. 15, 953-966.

Sammon, J.W., 1969. A nonlinear mapping for data structure analysis. IEEE Trans. Comput., C 18 (5), 401-409.

Torgerson, W., 1952. Multidimensional scaling: I. Theory and methods. Psychometrica 17, 401-419.

Venna, J., Kaski, S., 2001. Neighborhood preservation in nonlinear projection methods: an experimental study. Proc. Int. Conf. on Artificial Neural Networks (ICANN 2001). Springer, Vienna, Austria, pp. 485-491.

Vesanto, J., Himberg, J., Alhoniemi, E., Parhankangas, J., 2000. SOM Toolbox for Matlab 5. Report A57. Helsinki University of Technology, Neural Networks Research Centre, Espoo, Finland.

Vigário, R., Jousmäki, V., Hämäläinen, M., Hari, R., Oja, E., 1998. Independent component analysis for identification of artifacts in magnetoencephalographic recordings. Adv. Neural Inf. Process. Syst., vol. 10. MIT Press, Cambridge, MA, pp. 229-235.

Vigário, R., Särelä, J., Jousmäki, V., Hämäläinen, M., Oja, E., 2000. Independent component approach to the analysis of EEG and MEG recordings. IEEE Trans. Biomed. Eng. 47 (5), 589-593.

Ware, C., 2000. Information Visualization: Perception for Design. Morgan Kaufmann, San Francisco. 